\title{
Effects of Sinogram Filtering in the Quality of PET Reconstructions: Preliminary Results
}

\author{
M Abella, S. Redondo, J. J. Vaquero, Senior Member, IEEE, J. Sanchez-Gonzalez, M. Desco
}

\begin{abstract}
Low-pass filtering sinograms prior to reconstruction is a general practice to reduce noise. Sinograms are generally filtered in the radial direction, although other filtering schemes have been proposed.

It is known that the Fourier transform of a sinogram shows a particular shape of the spectral energy distribution ("bow-tie"). In this work, this property has been exploited to perform an adapted filter, whose performance has been compared with previously reported methods: angular, axial and stackgram domain filtering.

Stackgram and angular filtering degraded resolution ( $\sim 16$ and $\sim 5.7 \%$ respectively) while no significant enhancement in contrast to noise ratio (CNR) was achieved. Angular filtering resulted in a circle blurring artifact dependant on the distance to the center of the FOV.

Bow-tie filtering showed the best results (enhancement of $\sim \mathbf{2 6} \%$ in resolution and of $\sim \mathbf{1 2 \%}$ in CNR). Axial filtering degraded resolution but enhanced CNR $(\sim 14 \%)$, appearing as a good strategy to reduce radial filtering. Experiments on rodent images showed a noticeable image quality enhancement achieved when using bow-tie filtering combined with radial and axial filters.
\end{abstract}

\section{INTRODUCTION}

$\mathrm{D}$ ATA acquired in Positron Emission Tomography systems usually suffer from poor statistics, resulting in very noisy data that reconstruction algorithms have to deal with. In the classical reconstruction algorithm, filtered backprojection (FBP), noise reduction is achieved by the inclusion of a low-pass filter usually in the sinogram radial direction, though this filtering also degrades resolution. There have been several works studying the effect of different strategies for noise reduction. Daube-Witherspoon et al. studied the effect of angular and axial filtering on noise and resolution $[1,2]$. One of the results from their work was the non uniform blurring produced by the angular filter. In an attempt to address this problem, Stearns et al. proposed a non-stationary filter in the angular direction [3].

Happonen et al. proposed a new sinogram decomposition defining a new space for filtering, the so-called stackgram domain $[4,5]$. In this domain, data are represented as a volume where each slice is the back-projection of a row of the sinogram (fig. 1). This space enables filtering each point in the

Manuscript received November 17, 2006. This work was supported in part by from Ministerio de Educación y Ciencia (FIS-PI052583 and TEC200407052-C02), Ministerio de Industria (CD-TEAM, CENIT program) and Comunidad de Madrid (GR/SAL/024104).

All the authors are with the Unidad de Medicina Experimental, Hospital Gregorio Marañón, Madrid, CO 28007 Spain (telephone: 3491426 5067, web: www.hggm.es/image). image following its sinusoidal trajectory in the sinogram, if the filtering is done in the vertical direction (across the slices). This filtering scheme is claimed to prevent from blurring artifacts both in radial and angular dimensions.
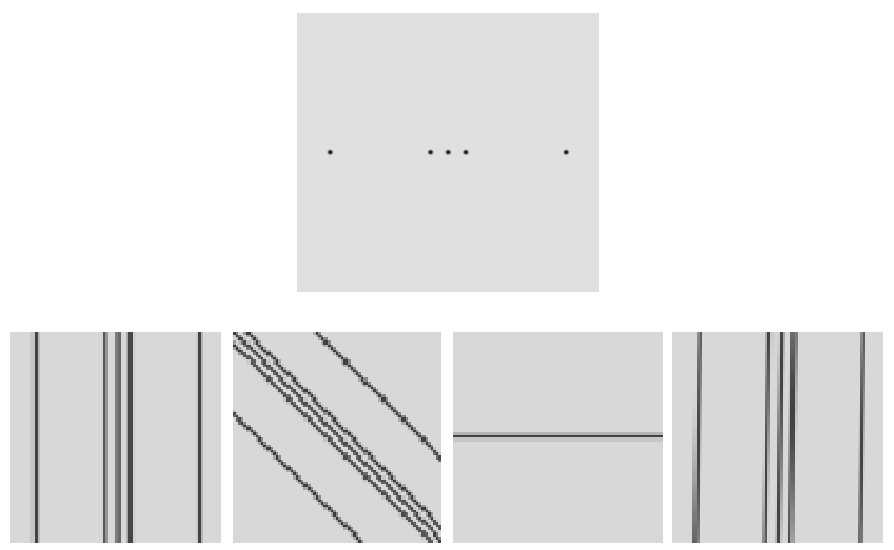

Fig. 1. Phantom with five point sources (top) and slices 0, 45, 90 y 179 in the stackgram domain.

A different approach is based on the work of Rattey and Lindgren [6] who studied the 2D Fourier transform of the sinogram in order to find its optimum sampling. These authors observed that the support region of the frequency content of any sinogram conformed what they called a "bow-tie" shape (fig. 2). This property, apart from being the key of the Fourier rebinning algorithm (FORE), was exploited for designing an optimum filter in [7].

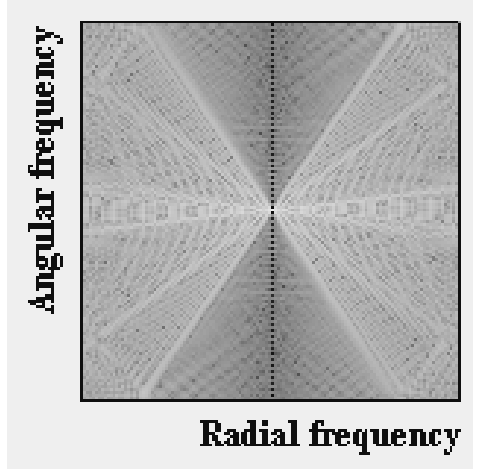

Fig. 2. 2D Fourier transform of a sinogram

The bow-tie support in the $\rho$ axis is limited by the highest frequency in the image, $\mathrm{w}_{\mathrm{u}}$ (limited by the acquisition process). In the $\theta$ axis, the limit depends on the radius of the object, $\mathrm{R}_{\mathrm{M}}$, according to the expression $\mathrm{R}_{\mathrm{M}}{ }^{*}\left|\mathrm{w}_{\mathrm{u}}\right|+1$. 
The objective of this work is to exploit the bow-tie property to perform an adapted filtering and to compare the results in terms of resolution and contrast to noise ratio with the standard techniques that involve just a stationary 1D filtering of the sinogram (radial, angular and axial) and with the filtering in the stackgram domain.

\section{MATERIAL AND METHODS}

\section{A. Phantom acquisition}

Measurements were made on the Image Quality Phantom developed by the NEMA animal PET Scanner Standard Task Force [8] and built by Dr. Richard Laforest from the Washington University in Saint Louis (fig. 3). The top part of the phantom is comprised of a cylinder of 19,5 $\mathrm{ml}$ with two cylinders inside of $0,75 \mathrm{ml}$ each. One of the inside cylinders is cold and the other was filled with approximately four times more activity concentration $(\mathrm{mCi} / \mathrm{ml})$ than the background cylinder. The phantom was placed with its axis parallel to the scanner axis.

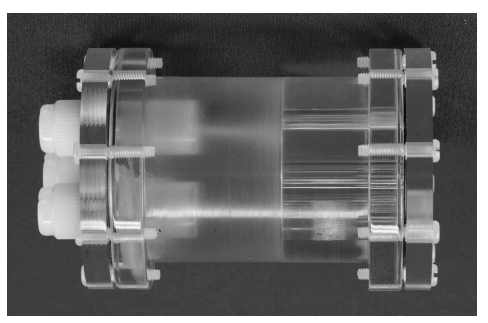

Fig. 3. Image Quality Phantom used for resolution and contrast to noise ration measurement.

Reconstructed image (fig. 4) shows that the radius of the phantom is approximately $75 \%$ of the radius of the total FOV.
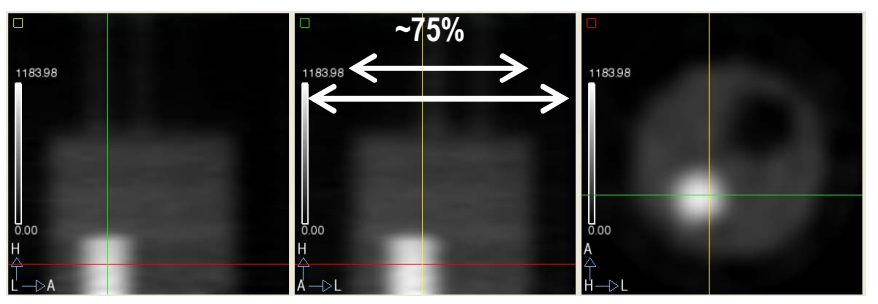

Fig. 4. Coronal, sagittal and axial views of the reconstructed volume. Phantom radius is approximately $75 \%$ of the radius of the total FOV.

\section{B. Data acquisition and reconstruction}

An acquisition of $20 \mathrm{~min}$. was carried out on the rPET scanner, SUINSA Medical Systems [9, 10]. This system has four detectors arranged as two orthogonal pairs (fig. 5).

Each detector is comprised of an array of 30x30 MLS crystals $\left(1.5 \times 1.5 \times 12 \mathrm{~mm}^{3}\right)$ optically coupled to a Hamamatsu H8500 flat-panel PS PMT. The system rotates $180^{\circ}$ covering a total angle of $194.4^{\circ}$. List data are rearranged into $180^{\circ}$ sinograms with 120 angular and 55 radial bins. The sinogram spacing is $0.8 \mathrm{~mm}$ and $1.5^{\circ}$ in the radial and angular directions, respectively.
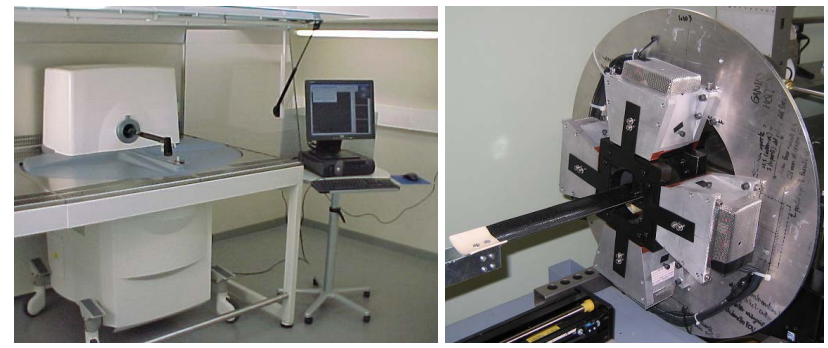

Fig. 5. rPET scanner commercialized by SUINSA Medical Systems

The data were reconstructed with a Single Slice Rebinning algorithm (SSRB) followed by a filtered back-projection. A Butterworth filter with order 12 at different cut-off frequencies was applied in the radial direction. The pixel size of the resulting image was $0.8 \mathrm{~mm}$ isotropic. In addition, each of the filters to be compared was applied: bow-tie, stackgram, angular and axial.

\section{Bow-tie filter implementation}

Bow-tie filter, M, was based on a binary mask defined by the following expression:

$M\left(w_{r}, w_{\theta}\right)=\operatorname{rect}\left(w_{r}, w_{\max }\right) * \operatorname{rect}\left(w_{\theta},\left[\left|w_{r}\right| \cdot \xi+1\right]\right)$

where:

$\operatorname{rect}(x, a)=\left\{\begin{array}{lr}1, & |x| \leq a \\ 0, & \text { otherwise }\end{array}\right.$

$\left\{\begin{array}{c}\omega_{r} \in[-1,1], \omega_{\theta} \in\left[-\frac{\pi}{2}, \frac{\pi}{2}\right] \\ \xi=\frac{R_{M}}{\text { FOV_radius }} \%\end{array}\right.$

For this work, we tested two bow-tie filters with values of parameter $\xi$ of $100 \%$ and $75 \%$ since the radius of the phantom is approximately the $75 \%$ of the radius of the total FOV (fig. 5). A five-point smoothing kernel was applied to the mask to avoid ringing artifacts (fig. 6).

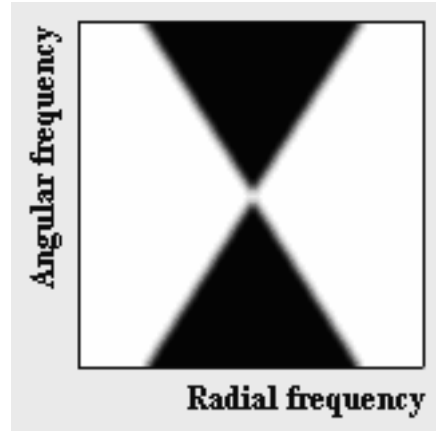

Fig. 6: Bow-tie mask in FT domain.

\section{Other filters}

The other filters were implemented as follows: 
- Radial filtering: Butterworth filter, with order 12 and variable cut-off frequency.

- Axial filtering: Gaussian mask of FWHM of $1.6 \mathrm{~mm}$.

- Angular filtering: Box-shape mask of 7.5 degrees.

- Stackgram domain: Box-shape mask of $1.6 \mathrm{~mm}$.

The size all of these filters was selected experimentally in order to achieve a similar performance in noise reduction.

\section{E. Contrast to noise ratio measurement}

To evaluate the effect of the different filters on the emission image noise we defined three volume masks by simple thresholding (fig. 7).
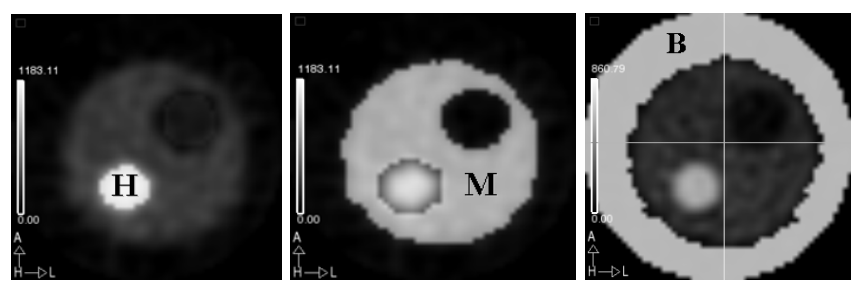

Fig. 7. Volume ROIs defined by thresholding for contrast to noise ration measurement.

Contrast to noise ratio (CNR) was then measured as follows ( $\sigma$ : standard deviation, $\mu$ : mean):

$$
C N R=\frac{\mu_{H}-\mu_{M}}{\sigma_{B}}
$$

\section{F. Resolution measurement}

We evaluated the effect of the different filters on the image resolution using the Edge Response Function (ERF). We took a ROI of $5 \times 70$ pixels on the edge of an image obtained by adding two slices of the phantom, followed by a interpolation to $220 \times 220$ pixels $(0.2 \mathrm{~mm}$ pixel size $)$, as it is shown in the left panel of fig. 8 .
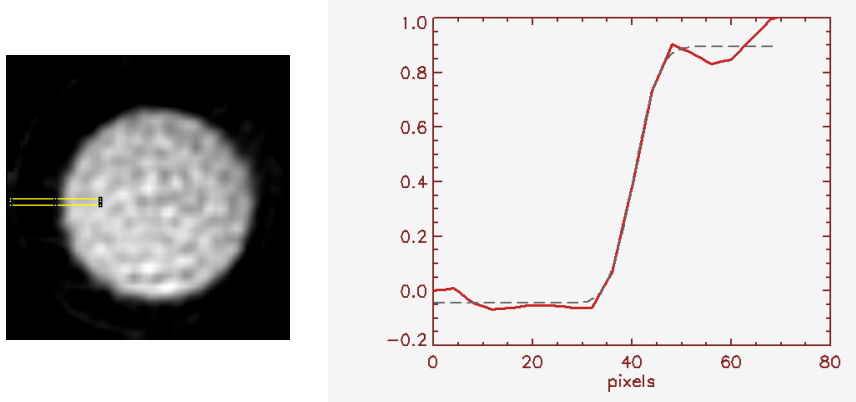

Fig. 8. Sum of two slices interpolated to $220 \times 220$ pixels where the profile is taking using a ROI of 5 high and 70 pixels long (left). Edge response function and the fitted function corresponding to the integral of a Gaussian (right).

FWHM has been measured by fitting the edge profile to the integral of a Gaussian distribution (fig. 8, right) according to the following expression:

$$
\operatorname{ERF}(x)=\int_{-\infty}^{x} \operatorname{PSF}(x)=\int_{-\infty}^{x} C \exp \left[\frac{(t-\mu)}{2 \sigma}\right] d t
$$

\section{RESULTS}

Fig. 9 shows the results in terms of CNR. The "Only radial" column corresponds to the result when applying only a radial filter with cut-off frequency of $80 \%$ of Nyquist. The rest of the columns represent the results when adding each of the filters to the previous reconstruction. There is no significant change in CNR when angular and stackgram filters were applied, while an increase of $12 \%$ when applying a bow-tie filter is achieved.

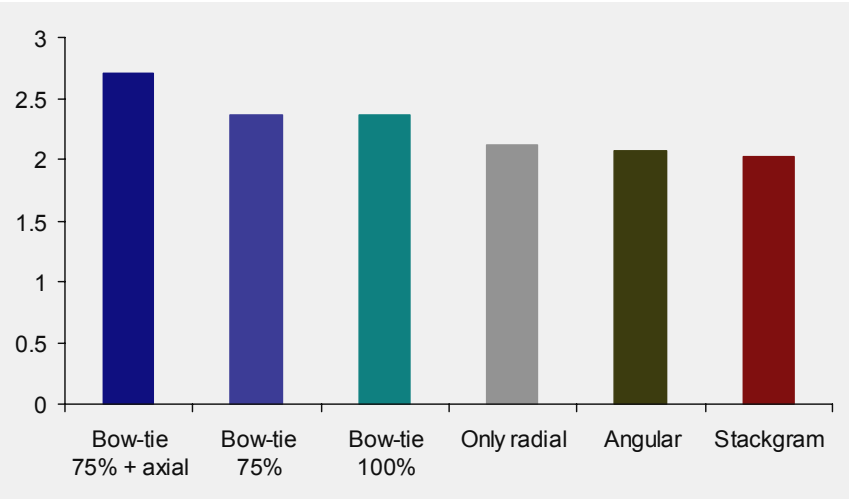

Fig. 9: CNR obtained when radial filtering (Butterworth, order:12, cut-off frequency: $80 \%$ Nyquist) is combined with different filters in each column.

Fig. 10 shows the results in terms of resolution. The "Only radial filt." line is the result of applying only the radial filter at different cut-off frequencies. The other lines are the result when adding each one of the filters we are comparing. There is a loss in resolution when applying angular and stackgram filters of 5.7 and $16 \%$ respectively. The use of a bow-tie filter for the total FOV $(\xi=100 \%)$ resulted in an enhancement of $20 \%$ in resolution. The use of a bow-tie mask adjusted to the size of the phantom $(\xi=75 \%)$ provided even better results $(26 \%)$.

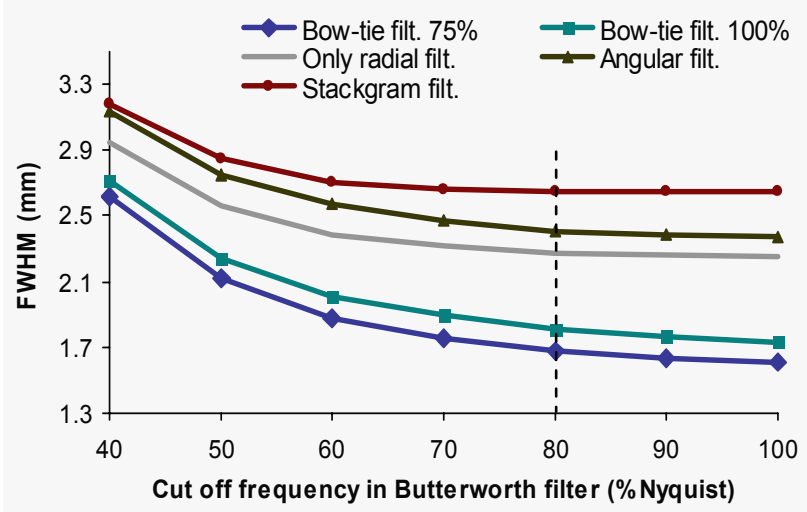

Fig. 10: Spatial resolution (FWHM). Radial filtering (Butterworth, order:12, variable cut-off frequency) is combined with different filters. 
The results of filtering in the axial direction are shown in fig. 11. This filtering produces a degradation in resolution while improving the CNR.
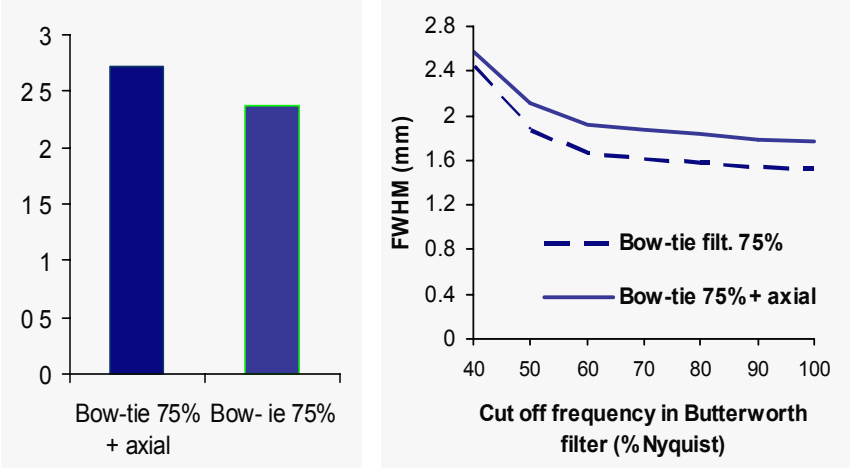

Fig. 11: Results when applying bow-tie filtering alone and combined with an axial filter in terms of CNR (left) and resolution (right).

As it had been previously reported in several works $[1-3,7]$ angular filtering resulted in a non uniform blurring dependant on the distance to the center of the field-of-view (fig. 12).

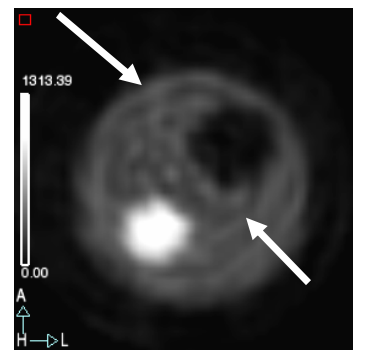

Fig. 12: Circular blurring due to angular filtering.

Experiments on rodent images demonstrated the enhancement achieved when using bow-tie filter combined with radial and axial filters (fig. 13). Figure 14 shows a profile along the white line in the axial view in fig. 13. A better pickto-valley ratio can be observed.

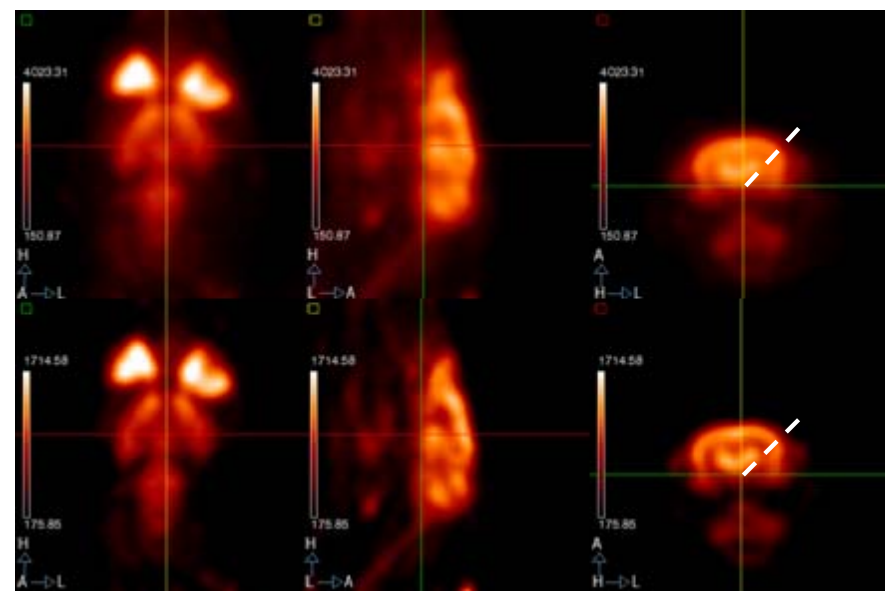

Fig. 13: FDG rat study. Coronal, sagittal and axial views. Axial filter: [0.2, $0.6,0.2]$. Radial filter: Butterworth, order 12, cut off freq. $70 \%$ Nyquist (top) Adding bow-tie filter with $\xi=50 \%$ (bottom).

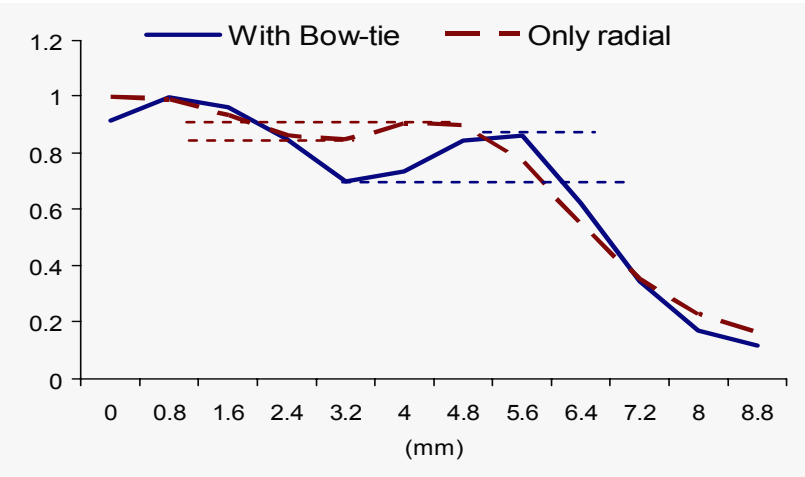

Fig. 14. Normalized profiles corresponding to the white line in fig. 13.

\section{Discussion}

A comparison of different methods for noise reduction in sinograms is presented.

Stackgram and angular filters seem to yield the poorer results in terms of resolution while no significant enhancement in CNR is achieved. Angular filtering results in a noticeable circle blurring dependant on the distance to the center of the FOV. The loss of resolution produced by the stackgram filter could be explained by the two interpolations implied in the back and forward projection (linear, in this work). A better interpolation scheme could provide better results. The problem of this filtering scheme is its computational cost (five times higher than any of the other methods).

Axial filtering degrades resolution but enhances CNR.

Bow-tie filtering increases both resolution and contrast to noise ratio. The promising results with the bow-tie filtering were obtained with a basic implementation of this filter: a windowed binary mask.

It would be advisable to investigate the possible dependence of the performance of the bow-tie filter on the frequency and statistical content image content.

Another important issue to be further studied is the effect of this filtering on the quantitative properties of the resulting image.

Experiments on rodent images showed a noticeable image quality enhancement achieved when using bow-tie filtering combined with radial and axial filters.

\section{REFERENCES}

[1] M. E. Daube-Witherspoon and R. E. Carson, Investigation of angular and axial smoothing of PET data, IEEE Nuc. Sci. Symp. Med. Im. Conf. 1996.

[2] M. E. Daube-Witherspoon and R.E. Carson, Investigation of angular smoothing of PET data, IEEE Trans. on Nuclear Science, Dec. 1997. 44(6): p. 2494-2499.

[3] C. W. Stearns, Context-sensitive angular filtering of PET transmission data. in IEEE Conf. Record of the Nuclear Science Symposium and Medical Imaging Conference. 1995.

[4] A. P. Happonen and S. Alenius. Sinogram filtering using a stackgram domain. in Proc. of the Second IASTED International Conference: Visualization, Imaging and Image Processing. 2002. Malaga, Spain. 
[5] A. P. Happonen and S. Alenius, A Comparison of Sinogram and Stackgram Domain Filtering Methods Employing L-Filters for Noise Reduction of Tomographic Data.

[6] P. A. Rattey and A.G. Lindgren, Sampling the 2-D Radon transform. IEEE Trans. on Acoustics, Speech and Signal Proc., Oct. 1981. 29(5): p. 994-1002.

[7] B. I. Andía, Nosstationary and nonlinear sinogram filtering for tomographic image reconstruction, in Department of Electrical Engineering,. June 2003, Notre Dame: Indiana.
[8] http://www.hggm.es/animalpet/, last date of access: 05-10-06.

[9] J. J. Vaquero, A. Molins, J. Ortuño, J. Pascau, M. Desco, Preliminary results of the small animal rotational positron emission tomography scanner. in Molecular Imaging and Biology 6(2): 102. 2004.

[10] J. J. Vaquero, E. Lage, L. Ricón, M. Abella, E. Vicente, M. Desco, rPET Detectors Design and Data Processing. IEEE Nuclear Science Symposium Conference Record, 2005. 